\title{
Castleman disease and paraneoplastic pemphigus
}

\author{
Else N. Kop MD, Marius A. MacKenzie MD PhD
}

Previously published at www.cmaj.ca

A

29-year-old woman presented with a six-month history of painful oral and vulvar erosions, and itchy erythematosquamous eroded papules grouped at multiple locations (Figures 1A and 1B). Apart from fatigue, she had no other symptoms. An initial diagnosis of pemphigus vulgaris was made and the patient was given immunosuppressive therapy for five months (i.e., low-dose prednisone for one month, then cyclosporine $200 \mathrm{mg}$ daily for four months). She did not improve and was referred to our hospital for a second opinion. We administered high-dose prednisone (90 mg daily) initially, and the skin lesions improved somewhat.

A biopsy of the skin from the pubic region showed intraepidermal blisters and depositions of IgG. Serologic testing showed antibodies directed against desmoglein 3 and plakins, which are indicative of paraneoplastic pemphigus. A chest radiograph showed a right posterior mediastinal mass (Figures 1C and 1D), which was resected.

Pathologic analysis of the resected mediastinal tumour showed a hyaline vascular type of unicentric Castleman disease, a well-known disorder associated with paraneoplastic pemphigus. After the surgery, all of the skin-related symptoms resolved and did not recur when prednisone was stopped three months later. Two years later, the patient has remained well.

Unicentric Castleman disease is a rare, isolated, benign lymphoproliferative disorder occurring in young adults. The disease is characterized by localized enlargement of hyperplastic lymph nodes in the mediastinum. The vast majority of patients are asymptomatic, and the disease is often an incidental finding in imaging studies. Complete surgical excision is curative, with a five-year rate of survival of $100 \%$. Nevertheless, follow-up is recommended because the tumours may recur and the risk of lymphoma is slightly increased. The prognosis is excellent, even for patients whose lesions cannot be completely resected. ${ }^{1}$

Paraneoplastic pemphigus is fairly uncommon. Fifteen percent of instances are caused by Castleman disease. Other associated neoplasias are non-Hodgkin lymphomas, chronic lymphatic leukemia, thymoma and Waldenström macroglobulinemia. ${ }^{2}$

From the Departments of Dermatology (Kop) and Hematology (MacKenzie), Radboud University Nijmegen Medical Centre, Nijmegen, Netherlands

CMAJ 2009. DOI:10.1503/cmaj.081504

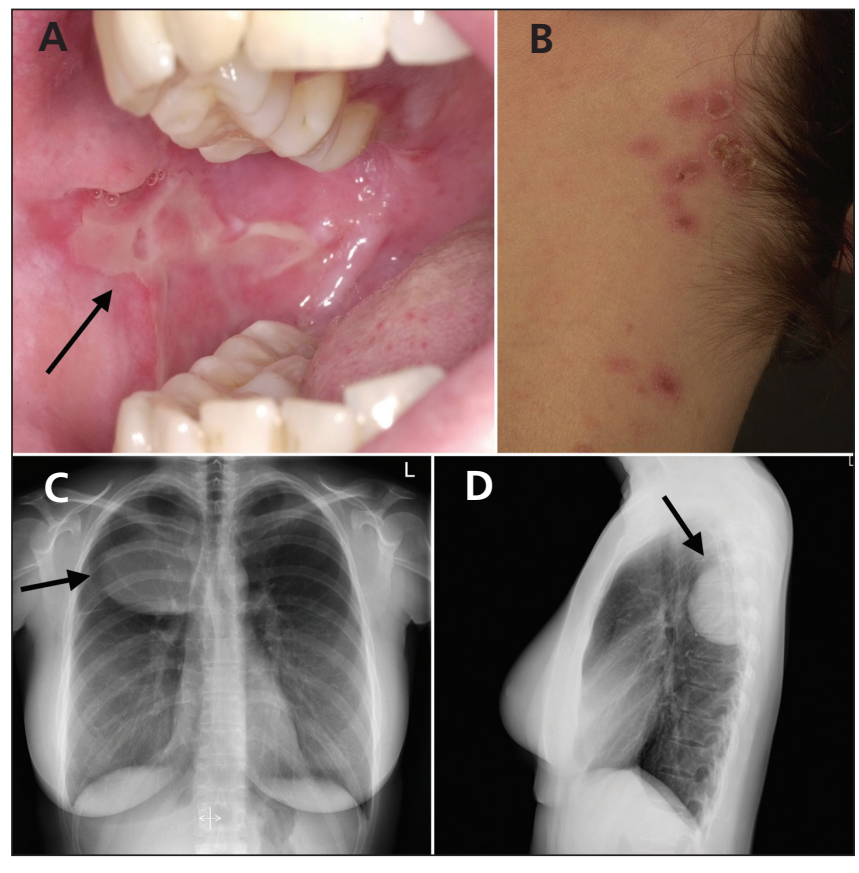

Figure 1: (A) Oral erosion (arrow) and (B) eroded papules on the neck of a patient with paraneoplastic pemphigus caused by Castleman disease. (C, D) Chest radiographs show a right posterior mediastinal mass (arrows).

This article has been peer reviewed.

Competing interests: None declared.

\section{REFERENCES}

1. Keller AR, Hochholzer L, Castleman B. Hyaline-vascular and plasma-cell types of giant lymph node hyperplasia of the mediastinum and other locations. Cancer 1972;29:670-83.

2. Ahmed AR, Avram MM, Duncan LM. Case records of the Massachusetts General Hospital. Weekly clinicopathological exercises. Case 23-2003. A 79-year-old woman with gastric lymphoma and erosive mucosal and cutaneous lesions. $N$ Engl J Med 2003;349:382-91.

Clinical images are chosen because they are particularly intriguing, classic or dramatic. Submissions of clear, appropriately labelled high-resolution images must be accompanied by a figure caption and the patient's written consent for publication. A brief explanation (300 words maximum) of the educational significance of the images with minimal references is required. 